International Journal of Pure and Applied Mathematics

Volume 87 No. 4 2013, 629-644

ISSN: 1311-8080 (printed version); ISSN: 1314-3395 (on-line version)

url: http://www.ijpam.eu

doi: http://dx.doi.org/10.12732/ijpam.v87i4.12

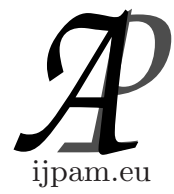

\title{
SIMULATION SUPPLEMENT TO GOODNESS-OF-FIT \\ TESTS DERIVED FROM CHARACTERIZATIONS OF CONTINUOUS DISTRIBUTIONS VIA RECORD VALUES
}

Dominik Szynal $^{1} \S$, Waldemar Wołyński ${ }^{2}$

${ }^{1}$ Department of Economics

Wydział Zamiejscowy KUL

ul. Ofiar Katynia 6, 37-450, Stalowa Wola, POLAND

${ }^{2}$ Faculty of Mathematics and Computer Science

Adam Mickiewicz University

ul. Umultowska 87, 61-614, Poznań, POLAND

\begin{abstract}
We give simulations of powers of goodness-of-fit tests for normality derived from characterizations of continuous distributions via record values and presented in Morris and Szynal [3].
\end{abstract}

AMS Subject Classification: 62G10, 62G30

Key Words: order statistics, record values, $U$-statistics, exponential and normal distributions, characterizations, goodness-of-fit tests, powers

\section{Introduction}

Morris and Szynal [3] constructed goodness-of-fit tests for continuous distributions using characterizations of continuous distributions via expected values of two functions of record values and $U$-statistics. In particular, tests for exponentiality, Rayleigh distribution and normality were presented. Simulations of powers of tests for exponential and Rayleigh distribution were also obtained to compare them with other tests. The aim of this contribution is to give simulations of powers of tests for normality to compare them with some recommended tests.

Received: July 10, 2013

(c) 2013 Academic Publications, Ltd.

$\S$ Correspondence author url: www.acadpubl.eu 
Tests and alternatives are taken from Pearson et al [4] as it was done in Morris and Szynal [2] where tests for normality are based on characterizations involving moments of order statistics.

Additionaly we choose for comparisons tests and alternatives studied in Kallenberg and Ledwina [1].

Simulations show that our tests for normality perform very well and can be applied instead of classical recommended tests.

\section{Tests for Normality}

Tests of $H: \quad X \sim N(\mu, \sigma)$ we will discuss are presented in Morris and Szynal [3]. Here by $f$ and $F$ we denote corresponding density and the cumulative distribution function, $\Lambda=\left\{\left(\mu, \sigma^{2}\right): \mu \in \mathbf{R}, \sigma^{2}>0\right\}$, i.e.

$$
\begin{aligned}
f\left(x ; \mu, \sigma^{2}\right) & =\frac{1}{\sigma \sqrt{2 \pi}} \exp \left(-\frac{1}{2 \sigma^{2}}(x-\mu)^{2}\right), \\
F(x) & =\int_{-\infty}^{x} f(t) d t, \quad-\infty<x<\infty .
\end{aligned}
$$

For $X \sim N(0,1)$ we write

$$
\phi(x)=\frac{1}{\sqrt{2 \pi}} \exp \left(-\frac{x^{2}}{2}\right), \quad \Phi(x)=\frac{1}{\sqrt{2 \pi}} \int_{-\infty}^{x} e^{-t^{2} / 2} d t .
$$

Moreover, we use

$$
\hat{\mu}_{n}=\bar{X}_{n}=\frac{1}{n} \sum_{i=1}^{n} X_{i}, \quad \hat{\sigma}_{n}^{2}=\frac{1}{n} \sum_{i=1}^{n}\left(X_{i}-\bar{X}_{n}\right)^{2}, \quad S_{n}=\sqrt{\hat{\sigma}_{n}^{2}} .
$$

The following quantities are appearing in the formulae of test-statistics:

$$
\begin{aligned}
a_{n}^{(r, k)}= & \frac{1}{\left(\begin{array}{l}
n \\
k
\end{array}\right)} \sum_{j=1}^{k-1}\left(\begin{array}{l}
k \\
j
\end{array}\right)\left(\begin{array}{l}
n-k \\
k-j
\end{array}\right)\left[2 \frac{\Gamma(2 r+2)}{k^{r}(k-j)^{r}} B_{\frac{k-j}{2 k-j}}(r+1, r+1)\right. \\
+ & \left.j \frac{\Gamma(2 r+1)}{(2 k-j)^{2 r+1}}-\frac{\Gamma^{2}(r+1)}{k^{2 r}}\right]+\frac{1}{\left(\begin{array}{l}
n \\
k
\end{array}\right)}\left[\frac{\Gamma(2 r+1)-\Gamma^{2}(r+1)}{k^{2 r}}\right], \\
b_{n}^{(r, k)=} & \frac{1}{\left(\begin{array}{l}
n \\
k
\end{array}\right)} \sum_{j=1}^{k-1}\left(\begin{array}{l}
k \\
j
\end{array}\right)\left(\begin{array}{l}
n-k \\
k-j
\end{array}\right)\left[2(2 k-j) \frac{\Gamma(2 r+2)+\Gamma(2 r+3)}{k^{r+1}(k-j)^{r+1}}\right. \\
& \left.\quad B_{\frac{k-j}{2 k-j}}(r+2, r+2)+j \frac{\Gamma(2 r+2)}{(2 k-j)^{2 r+2}}-\frac{\Gamma(r+1) \Gamma(r+2)}{k^{2 r+1}}\right]
\end{aligned}
$$




$$
\begin{aligned}
& +\frac{1}{\left(\begin{array}{l}
n \\
k
\end{array}\right)}\left[\frac{\Gamma(2 r+2)-\Gamma(r+1) \Gamma(r+2)}{k^{2 r+1}}\right] \\
c_{n}^{(r, k)} & =\frac{1}{\left(\begin{array}{l}
n \\
k
\end{array}\right)} \sum_{j=1}^{k-1}\left(\begin{array}{l}
k \\
j
\end{array}\right)\left(\begin{array}{l}
n-k \\
k-j
\end{array}\right)\left[2 \frac{\Gamma(2 r+4)}{k^{r+1}(k-j)^{r+1}} B_{\frac{k-j}{2 k-j}}(r+2, r+2)\right. \\
& \left.+j \frac{\Gamma(2 r+3)}{(2 k-j)^{2 r+3}}-\frac{\Gamma^{2}(r+2)}{k^{2 r+2}}\right]+\frac{1}{\left(\begin{array}{l}
n \\
k
\end{array}\right)}\left[\frac{\Gamma(2 r+3)-\Gamma^{2}(r+2)}{k^{2 r+2}}\right],
\end{aligned}
$$

where

$$
B_{x}(\alpha, \beta)=\int_{0}^{x} t^{\alpha-1}(1-t)^{\beta-1} d t, \quad 0<x<1 ; \quad \alpha, \beta>0,
$$

denotes the incomplete beta function.

$$
\begin{aligned}
& E_{1}^{(r, k)}=\mathrm{E}\left[\phi(Z)(1-\Phi(Z))^{k-2} \log ^{r-1} \frac{1}{1-\Phi(Z)}\right], \quad Z \sim N(0,1), \\
& E_{2}^{(r, k)}=\mathrm{E}\left[Z \phi(Z)(1-\Phi(Z))^{k-2} \log ^{r-1} \frac{1}{1-\Phi(Z)}\right] . \\
& s_{n}^{(r, k)}=\frac{k^{2} r^{2}}{n}\left[\left(E_{1}^{(r, k)}\right)^{2}+\frac{1}{2}\left(E_{2}^{(r, k)}\right)^{2}\right] \\
& t_{n}^{(r, k)}=\frac{k^{2} r(r+1)}{n}\left[E_{1}^{(r, k)} E_{1}^{(r+1, k)}+\frac{1}{2} E_{2}^{(r, k)} E_{2}^{(r+1, k)}\right] \\
& u_{n}^{(r, k)}=\frac{k^{2}(r+1)^{2}}{n}\left[\left(E_{1}^{(r+1, k)}\right)^{2}+\frac{1}{2}\left(E_{2}^{(r+1, k)}\right)^{2}\right] \\
& a_{n 1}^{(r, k)}=a_{n}^{(r, k)}-s_{n}^{(r, k)} \\
& b_{n 1}^{(r, k)}=b_{n}^{(r, k)}-t_{n}^{(r, k)} \\
& c_{n 1}^{(r, k)}=c_{n}^{(r, k)}-u_{n}^{(r, k)} \\
& \Delta_{n 1}^{(r, k)}=\operatorname{det}\left[\begin{array}{l}
a_{n 1}^{(r, k)} b_{n 1}^{(r, k)}{ }_{n 1}^{(r, k)} \\
\left.c_{n 1}^{(r, k)}\right]
\end{array}\right.
\end{aligned}
$$

The proposed in Morris and Szynal [3] test-statistics for normality are as follows

$$
\begin{aligned}
\hat{T}_{n}^{(r, k)} & =\frac{1}{\Delta_{n 1}^{(r, k)}}\left[c _ { n 1 } ^ { ( r , k ) } \left(\frac{1}{\left(\begin{array}{l}
n \\
k
\end{array}\right)} \sum_{i=1}^{n-k+1}\left(\begin{array}{l}
n-i \\
k-1
\end{array}\right) \log ^{r} \frac{1}{1-\Phi\left(\frac{X_{i: n}-\bar{X}_{n}}{S_{n}}\right)}\right.\right. \\
& \left.-\frac{\Gamma(r+1)}{k^{r}}\right)^{2}
\end{aligned}
$$




$$
\begin{aligned}
& -2 b_{n 1}^{(r, k)}\left(\frac{1}{\left(\begin{array}{l}
n \\
k
\end{array}\right)} \sum_{i=1}^{n-k+1}\left(\begin{array}{l}
n-i \\
k-1
\end{array}\right) \log ^{r} \frac{1}{1-\Phi\left(\frac{X_{i: n}-\bar{X}_{n}}{S_{n}}\right)}-\frac{\Gamma(r+1)}{k^{r}}\right) \\
& \times \quad\left(\frac{1}{\left(\begin{array}{l}
n \\
k
\end{array}\right)} \sum_{i=1}^{n-k+1}\left(\begin{array}{l}
n-i \\
k-1
\end{array}\right) \log ^{r+1} \frac{1}{1-\Phi\left(\frac{X_{i: n}-\bar{X}_{n}}{S_{n}}\right)}-\frac{\Gamma(r+2)}{k^{r+1}}\right) \\
& +a_{n 1}^{(r, k)}\left(\frac{1}{\left(\begin{array}{l}
n \\
k
\end{array}\right)} \sum_{i=1}^{n-k+1}\left(\begin{array}{l}
n-i \\
k-1
\end{array}\right) \log ^{r+1} \frac{1}{1-\Phi\left(\frac{X_{i: n}-\bar{X}_{n}}{S_{n}}\right)}-\frac{\Gamma(r+2)}{k^{r+1}}\right) \\
& =\hat{T}_{n ; c_{1}}^{(r, k)}+\hat{T}_{n ; c_{2}}^{(r, k)}=\hat{T}_{n ; c_{3}}^{(r, k)}+\hat{T}_{n ; c_{4}}^{(r, k)},
\end{aligned}
$$

where

$$
\begin{aligned}
& \hat{T}_{n ; c_{1}}=\frac{1}{a_{n 1}^{(r, k)}}\left[\frac{1}{\left(\begin{array}{l}
n \\
k
\end{array}\right)} \sum_{i=1}^{n-k+1}\left(\begin{array}{l}
n-i \\
k-1
\end{array}\right) \log ^{r} \frac{1}{1-\Phi\left(\frac{X_{i: n}-\bar{X}_{n}}{S_{n}}\right)}-\left.\frac{\Gamma(r+1)}{k^{r}}\right|^{2}\right. \\
& \hat{T}_{n ; c_{2}}=\frac{1}{\Delta_{n 1}^{(r, k)} a_{n 1}^{(r, k)}}\left[a_{n 1}^{(r, k)} \frac{1}{\left(\begin{array}{l}
n \\
k
\end{array}\right)} \sum_{i=1}^{n-k+1}\left(\begin{array}{l}
n-i \\
k-1
\end{array}\right) \log ^{r+1} \frac{1}{1-\Phi\left(\frac{X_{i: n}-\bar{X}_{n}}{S_{n}}\right)}\right. \\
& -b_{n 1}^{(r, k)} \frac{1}{\left(\begin{array}{l}
n \\
k
\end{array}\right)} \sum_{i=1}^{n-k+1}\left(\begin{array}{l}
n-i \\
k-1
\end{array}\right) \log ^{r} \frac{1}{1-\Phi\left(\frac{X_{i: n}-\bar{X}_{n}}{S_{n}}\right)} \\
& \left.-\left(a_{n 1}^{(r, k)} \frac{\Gamma(r+2)}{k^{r+1}}-b_{n 1}^{(r, k)} \frac{\Gamma(r+1)}{k^{r}}\right)\right]^{2} \\
& \hat{T}_{n ; c_{3}}=\frac{1}{c_{n 1}^{(r, k)}}\left[\frac{1}{\left(\begin{array}{l}
n \\
k
\end{array}\right)} \sum_{i=1}^{n-k+1}\left(\begin{array}{l}
n-i \\
k-1
\end{array}\right) \log ^{r+1} \frac{1}{1-\Phi\left(\frac{X_{i: n}-\bar{X}_{n}}{S_{n}}\right)}-\left.\frac{\Gamma(r+2)}{k^{r+1}}\right|^{2}\right. \\
& \hat{T}_{n ; c 4}=\frac{1}{\Delta_{n 1}^{(r, k)} c_{n 1}^{(r, k)}}\left[c_{n 1}^{(r, k)} \frac{1}{\left(\begin{array}{l}
n \\
k
\end{array}\right)} \sum_{i=1}^{n-k+1}\left(\begin{array}{l}
n-i \\
k-1
\end{array}\right) \log ^{r} \frac{1}{1-\Phi\left(\frac{X_{i: n}-\bar{X}_{n}}{S_{n}}\right)}\right. \\
& -b_{n 1}^{(r, k)} \frac{1}{\left(\begin{array}{l}
n \\
k
\end{array}\right)} \sum_{i=1}^{n-k+1}\left(\begin{array}{l}
n-i \\
k-1
\end{array}\right) \log ^{r+1} \frac{1}{1-\Phi\left(\frac{X_{i: n}-\bar{X}_{n}}{S_{n}}\right)} \\
& \left.-\left(c_{n 1}^{(r, k)} \frac{\Gamma(r+1)}{k^{r}}-b_{n 1}^{(r, k)} \frac{\Gamma(r+2)}{k^{r+1}}\right)\right]^{2} \text {. }
\end{aligned}
$$




\section{Simulation Results}

For an empirical comparison of the performances of our tests we use some alternatives and tests choosing from Pearson et al [4] with numbering using there (cf. Table 1) and studied in Morris and Szynal [2]. Here we do also comparison of the performances of our tests with the data driven smooth tests of Kallenberg and Ledwina [1] (cf. Table 2).

We discuss the following omnibus tests:

$K^{2}$ : The Brown \& Shenton test,

$R$ : The rectangle test,

$S-W$ : The Shapiro-Wilk test ( $W$ in [4]),

$Y:$ The D'Agostino test,

$W:$ The Shapiro-Wilk test ( $W$ in [1]),

$W_{S}$ : The data driven smooth test statistic,

$W_{S 1}$ : The data driven smooth modified test statistic,

$W_{S 2}$ : The data driven smooth test statistic without "adjustment".

When $n=20$ and $n=50$ the powers of the test $\hat{T}_{n}^{(r, k)}$ and their components $\hat{T}_{n ; c_{1}}^{(r, k)}, \hat{T}_{n ; c_{2}}^{(r, k)}, \hat{T}_{n ; c_{3}}^{(r, k)}, \hat{T}_{n ; c_{4}}^{(r, k)}$ were investigated for $r=-0.499,-0.45,-0.4,-0.1$, $0.1,0.3,0.5,0.7,0.9,1.0,1.1,1.3,1.5,1.7,2.0$ and $k=1,2,3,4,5$. Critical values were simulated using 100000 samples and the associated power were obtained using 100000 samples, but only some results are presented here.

The alternatives chosen in Morris and Szynal [2] we write Alt.M-S while the alternatives used in Kallenberg and Ledwina [1] we denote Alt.K-L.

For samples of size $n=20$ we include simulations for some favorable omnibus tests under symmetric alternatives with Av. powers $\geq 34.0$ (Alt.M-S); $\geq 37$ (Alt.K-L) and under skew alternatives with Av. powers $\geq 50.0$ (Alt.M-S); $\geq 44$ (Alt.K-L) (Tables 3-6).

For samples of size $n=50$ we include simulations for some favorable omnibus tests under symmetric alternatives with Av. powers $\geq 62.0$ (Alt.M-S); $\geq 69$ (Alt.K-L) and under skew alternatives with Av. powers $\geq 83.0$ (Alt.M-S); $\geq 73$ (Alt.K-L) (Tables 7-10). 


\begin{tabular}{|c|c|c|c|c|c|c|c|c|c|c|c|}
\hline \multicolumn{6}{|c|}{$n=20$} & \multicolumn{6}{|c|}{$n=50$} \\
\hline \multicolumn{6}{|c|}{ symmetric } & \multicolumn{6}{|c|}{ symmetric } \\
\hline & Alt.M-S & $K^{2}$ & $R$ & $\overline{S-W}$ & $\bar{Y}$ & & Alt.M-S & $K^{2}$ & $R$ & $\overline{S-W}$ & $Y$ \\
\hline 1 & $S B(0 ; 0.5)$ & 38 & 40 & 48 & 8 & 1 & $S B(0 ; 0.5)$ & 96 & 96 & 100 & 43 \\
\hline 4 & $S B(0 ; 0.707)$ & 14 & 16 & 20 & 8 & 4 & $S B(0 ; 0.707)$ & 52 & 58 & 70 & 61 \\
\hline 7 & $\operatorname{Beta}(2 ; 2)$ & 14 & 4 & 2 & 7 & 7 & $\operatorname{Beta}(2 ; 2)$ & 18 & 17 & 23 & 32 \\
\hline 15 & Logistic(1) & 16 & 12 & 12 & 11 & 15 & Logistic(1) & 23 & 22 & 8 & 22 \\
\hline 18 & Laplace & 31 & 26 & 26 & 28 & 18 & Laplace & 54 & 54 & 39 & 56 \\
\hline 21 & $S C(0.1 ; 3)$ & 36 & 34 & 26 & 28 & 21 & $S C(0.1 ; 3)$ & 61 & 58 & 48 & 58 \\
\hline 24 & $S C(0.1 ; 5)$ & 61 & 60 & 50 & 48 & 24 & $S C(0.1 ; 5)$ & 88 & 88 & 80 & 86 \\
\hline 27 & $S C(0.05 ; 7)$ & 49 & 48 & 42 & 41 & 27 & $S C(0.05 ; 7)$ & 80 & 80 & 73 & 74 \\
\hline 30 & $t(4)$ & 25 & 24 & 25 & 26 & 30 & $t(4)$ & 46 & 43 & 36 & 49 \\
\hline 32 & $t(1)$ & 79 & 80 & 91 & 92 & 32 & $t(1)$ & 98 & 98 & 99 & 100 \\
\hline & Av. & 36.3 & 34.4 & 34.2 & 29.7 & & Av. & 61.6 & 61.4 & 57.6 & 58.1 \\
\hline \multicolumn{6}{|c|}{ skew } & \multicolumn{6}{|c|}{ skew } \\
\hline \multicolumn{2}{|r|}{ Alt.M-S } & $K^{2}$ & $R$ & $S-W$ & $\bar{Y}$ & \multicolumn{2}{|r|}{ Alt.M-S } & $K^{2}$ & $R$ & $S-W$ & $\bar{Y}$ \\
\hline 36 & $S B(0.533 ; 0.5)$ & 29 & 29 & 75 & 11 & 36 & $S B(0.533 ; 0.5)$ & 82 & 82 & 100 & 18 \\
\hline 38 & $\operatorname{Beta}(2 ; 1)$ & 12 & 11 & 29 & 6 & 38 & $\operatorname{Beta}(2 ; 1)$ & 32 & 31 & 91 & 12 \\
\hline 40 & $S B(1 ; 1)$ & 18 & 18 & 36 & 11 & 40 & $S B(1 ; 1)$ & 35 & 35 & 86 & 10 \\
\hline 42 & $L C(0.2 ; 5)$ & 43 & 43 & 86 & 48 & 42 & $L C(0.2 ; 5)$ & 92 & 94 & 100 & 84 \\
\hline 44 & Weibull(2) & 14 & 14 & 19 & 7 & 44 & Weibull $(2)$ & 33 & 32 & 38 & 12 \\
\hline 50 & $\chi_{4}^{2}$ & 40 & 39 & 46 & 24 & 50 & $\chi_{4}^{2}$ & 80 & 82 & 96 & 62 \\
\hline 52 & $L C(0.05 ; 5)$ & 53 & 51 & 59 & 52 & 52 & $L C(0.05 ; 5)$ & 90 & 89 & 82 & 82 \\
\hline 54 & $L C(0.05 ; 7)$ & 61 & 60 & 63 & 62 & 54 & $L C(0.05 ; 7)$ & 93 & 93 & 90 & 90 \\
\hline 56 & Weibull $((0.5)$ & 96 & 96 & 100 & 96 & 56 & Weibull $((0.5)$ & 100 & 100 & 100 & 100 \\
\hline 58 & $L N(0 ; 1)$ & 82 & 81 & 83 & 77 & 58 & $L N(0 ; 1)$ & 99 & 100 & 100 & 99 \\
\hline & Av. & 44.8 & 44.2 & 59.6 & 39.4 & & Av. & 73.6 & 73.8 & 88.3 & 56.9 \\
\hline
\end{tabular}

Table 1: (Source: Pearson et al [4]) Empirical comparision of the performances of the tests $K^{2}, R, S-W$ and $Y$ based on 200 replications. The entries are simulated powers of $5 \%$ tests. 


\begin{tabular}{|c|c|c|c|c|c|c|c|c|c|c|c|c|c|c|c|c|c|}
\hline \multicolumn{9}{|c|}{$n=20$} & \multicolumn{9}{|c|}{$n=50$} \\
\hline \multicolumn{9}{|c|}{ symmetric } & \multicolumn{9}{|c|}{ symmetric } \\
\hline & Alt.K-L & $W$ & $W_{\mathrm{S}}$ & $W_{\mathrm{S} 1}$ & $W_{\mathrm{S} 2}$ & $K^{2}$ & $R$ & $\bar{Y}$ & & Alt.K-L & $W$ & $W_{\mathrm{S}}$ & $W_{\mathrm{S} 1}$ & $W_{\mathrm{S}_{2}}$ & $K^{2}$ & $R$ & $Y$ \\
\hline 1 & $S B(0 ; 0.5)$ & 44 & 36 & 34 & 26 & 38 & 40 & 8 & 1 & $S B(0 ; 0.5)$ & 99 & 67 & 93 & 55 & 96 & 96 & 43 \\
\hline 2 & $T U(1.5)$ & 26 & 20 & 19 & 14 & 19 & 20 & 12 & 2 & $T U(1.5)$ & 92 & 34 & 74 & 26 & 78 & 80 & 58 \\
\hline 5 & $T U(0.7)$ & 12 & 11 & 9 & 8 & 10 & 12 & 12 & 5 & $T U(0.7)$ & 62 & 13 & 45 & 9 & 47 & 48 & 61 \\
\hline 15 & Logistic(1) & 12 & 10 & 13 & 11 & 16 & 12 & 11 & 15 & Logistic(1) & 13 & 13 & 21 & 12 & 23 & 22 & 22 \\
\hline 17 & $T U(10)$ & 82 & 82 & 87 & 85 & 46 & 45 & 84 & 17 & $T U(10)$ & 99 & 99 & 100 & 99 & 58 & 66 & 100 \\
\hline 20 & $S C(0.05 ; 3)$ & 19 & 17 & 19 & 16 & 24 & 22 & 20 & 20 & $S C(0.05 ; 3)$ & 31 & 25 & 38 & 24 & 44 & 40 & 38 \\
\hline 22 & $S C(0.2 ; 5)$ & 71 & 65 & 74 & 65 & 69 & 68 & 70 & 22 & $S C(0.2 ; 5)$ & 95 & 92 & 98 & 92 & 96 & 97 & 98 \\
\hline 25 & $S C(0.05 ; 5)$ & 36 & 33 & 37 & 32 & 39 & 38 & 34 & 25 & $S C(0.05 ; 5)$ & 62 & 55 & 66 & 55 & 71 & 70 & 64 \\
\hline 27 & $S C(0.05 ; 7)$ & 45 & 42 & 46 & 42 & 49 & 48 & 41 & 27 & $S C(0.05 ; 7)$ & 74 & 70 & 77 & 70 & 80 & 80 & 74 \\
\hline \multirow[t]{2}{*}{28} & $S U(0,1)$ & 43 & 36 & 47 & 38 & 44 & 42 & 42 & 28 & $S U(0,1)$ & 68 & 61 & 81 & 61 & 75 & 74 & 83 \\
\hline & Av. & 39.0 & 35.2 & 38.5 & 33.7 & 35.4 & 34.7 & 33.4 & & Av. & 69.5 & 52.9 & 69.3 & 50.3 & 66.8 & 67.3 & 64.1 \\
\hline \multicolumn{9}{|c|}{ skew } & \multicolumn{9}{|c|}{ skew } \\
\hline & Alt.K-L & $W$ & $W_{\mathrm{S}}$ & $W_{\mathrm{S} 1}$ & $W_{\mathrm{S} 2}$ & $K^{2}$ & $R$ & $Y$ & & Alt.K-L & $W$ & $W_{\mathrm{S}}$ & $W_{\mathrm{S} 1}$ & $W_{\mathrm{S} 2}$ & $K^{2}$ & $R$ & $Y$ \\
\hline 36 & $S B(0.533 ; 0.5)$ & 73 & 63 & 47 & 55 & 29 & 29 & 11 & 36 & $S B(0.533 ; 0.5)$ & 100 & 96 & 95 & 92 & 82 & 82 & 18 \\
\hline 40 & $S B(1 ; 1)$ & 31 & 29 & 17 & 29 & 18 & 18 & 11 & 40 & $S B(1 ; 1)$ & 81 & 72 & 57 & 71 & 35 & 35 & 10 \\
\hline 41 & $L C(0.2 ; 3)$ & 31 & 27 & 19 & 28 & 16 & 16 & 9 & 41 & $L C(0.2 ; 3)$ & 60 & 68 & 52 & 69 & 29 & 31 & 18 \\
\hline 44 & Weibull (2) & 15 & 15 & 10 & 16 & 14 & 14 & 7 & 44 & Weibull(2) & 41 & 41 & 29 & 41 & 33 & 32 & 12 \\
\hline 45 & $L C(0.1 ; 3)$ & 25 & 24 & 21 & 26 & 30 & 28 & 12 & 45 & $L C(0.1 ; 3)$ & 50 & 58 & 51 & 58 & 50 & 50 & 38 \\
\hline 46 & $\chi_{10}^{2}$ & 25 & 23 & 18 & 26 & 27 & 25 & 16 & 46 & $\chi_{10}^{2}$ & 57 & 61 & 48 & 62 & 46 & 46 & 20 \\
\hline 47 & $L C(0.05 ; 3)$ & 18 & 17 & 18 & 17 & 26 & 25 & 12 & 47 & $L C(0.05 ; 3)$ & 32 & 33 & 37 & 34 & 43 & 42 & 30 \\
\hline 48 & $L C(0.1 ; 5)$ & 76 & 72 & 72 & 73 & 64 & 63 & 59 & 48 & $L C(0.1 ; 5)$ & 98 & 97 & 98 & 97 & 97 & 98 & 96 \\
\hline 49 & $S U(-1 ; 2)$ & 22 & 19 & 20 & 21 & 20 & 20 & 18 & 49 & $S U(-1 ; 2)$ & 37 & 43 & 40 & 42 & 46 & 44 & 27 \\
\hline 50 & $\chi_{4}^{2}$ & 53 & 51 & 38 & 52 & 40 & 39 & 24 & 50 & $\chi_{4}^{2}$ & 95 & 94 & 86 & 93 & 80 & 82 & 62 \\
\hline 52 & $L C(0.05 ; 5)$ & 55 & 48 & 54 & 49 & 53 & 51 & 52 & 52 & $L C(0.05 ; 5)$ & 85 & 79 & 87 & 78 & 90 & 89 & 82 \\
\hline 54 & $L C(0.05 ; 7)$ & 65 & 64 & 65 & 63 & 61 & 60 & 62 & 54 & $L C(0.05 ; 7)$ & 92 & 91 & 92 & 90 & 93 & 93 & 90 \\
\hline 57 & $S U(1 ; 1)$ & 73 & 73 & 68 & 73 & 68 & 68 & 60 & 57 & $S U(1 ; 1)$ & 96 & 98 & 97 & 98 & 96 & 98 & 96 \\
\hline 58 & $L N(0 ; 1)$ & 94 & 91 & 85 & 92 & 82 & 81 & 77 & 58 & $L N(0 ; 1)$ & 100 & 100 & 100 & 100 & 99 & 100 & 99 \\
\hline & Av. & 46.9 & 44.0 & 39.4 & 44.3 & 39.1 & 38.4 & 30.7 & & Av. & 73.1 & 73.6 & 69.2 & 73.2 & 65.6 & 65.9 & 49.9 \\
\hline
\end{tabular}

Table 2: (Source: Kallenberg and Ledwina [1] and Pearson et al [4]) Empirical comparision of the performances of the tests $W, W_{S}, W_{S 1}$ and $W_{S 2}$ based on 10000 replications and $K^{2}, R$ and $Y$ based on 200 replications. The entries are simulated powers of $5 \%$ tests. 


\begin{tabular}{|c|c|c|c|c|c|c|c|c|c|}
\hline \multicolumn{10}{|c|}{$n=20$} \\
\hline \multicolumn{10}{|c|}{ symmetric } \\
\hline$k$ & & & & 1 & & & & & \\
\hline Tests & $\hat{T}_{n, c)}^{(r, k)}$ & $\hat{T}_{n ; c 4}^{(r, k)}$ & $\hat{T}_{n ; c 1}^{(r, k)}$ & $\hat{T}_{n ; c 4}^{(r, k)}$ & $\hat{T}_{n ; c,}^{(r, k)}$ & $\hat{T}_{n ; c 2}^{(r, k)}$ & $\hat{T}_{n ; c 2}^{(r, k)}$ & $\hat{T}_{n ; c 4}^{(r, k)}$ & $\hat{T}_{n}^{(r, k)}$ \\
\hline Alt.M-S/r & 0.3 & 0.3 & 0.7 & 0.7 & 0.9 & 1.0 & 1.1 & 0.5 & 0.5 \\
\hline 1 & 38 & 44 & 37 & 38 & 43 & 43 & 38 & 53 & 33 \\
\hline 4 & 17 & 19 & 17 & 17 & 18 & 19 & 17 & 28 & 11 \\
\hline 7 & 7 & 7 & 7 & 6 & 7 & 7 & 7 & 12 & 4 \\
\hline 15 & 13 & 13 & 13 & 13 & 13 & 13 & 13 & 10 & 14 \\
\hline 18 & 27 & 29 & 28 & 27 & 28 & 28 & 28 & 23 & 31 \\
\hline 21 & 30 & 31 & 31 & 30 & 31 & 32 & 31 & 25 & 32 \\
\hline 24 & 56 & 57 & 56 & 55 & 56 & 57 & 56 & 51 & 58 \\
\hline 27 & 46 & 46 & 46 & 46 & 46 & 46 & 46 & 43 & 47 \\
\hline 30 & 26 & 27 & 26 & 26 & 26 & 27 & 26 & 21 & 28 \\
\hline 32 & 85 & 86 & 86 & 85 & 85 & 86 & 85 & 84 & 88 \\
\hline Av. & 34.5 & 36.0 & 34.7 & 34.2 & 35.4 & 35.8 & 34.8 & 35.1 & 34.6 \\
\hline$k$ & & 2 & & & & 3 & & & \\
\hline Tests & $\hat{T}_{n ; c_{2}}^{(r, k)}$ & $\hat{T}_{n}^{(r, k)}$ & $\hat{T}_{n ; c_{2}}^{(r, k)}$ & $\hat{T}_{n}^{(r, k)}$ & $\hat{T}_{n}^{(r, k)}$ & $\hat{T}_{n}^{(r, k)}$ & $\hat{T}_{n ; c)}^{(r, k)}$ & $\hat{T}_{n}^{(r, k)}$ & \\
\hline Alt.M-S/r & 0.7 & 0.7 & 0.9 & 0.9 & 1.0 & 1.1 & 1.3 & 1.3 & \\
\hline 1 & 41 & 32 & 38 & 46 & 50 & 51 & 44 & 50 & \\
\hline 4 & 16 & 10 & 15 & 22 & 23 & 24 & 21 & 23 & \\
\hline 7 & 6 & 3 & 5 & 9 & 10 & 10 & 9 & 9 & \\
\hline 15 & 13 & 14 & 14 & 11 & 10 & 11 & 11 & 10 & \\
\hline 18 & 32 & 32 & 32 & 26 & 27 & 27 & 28 & 25 & \\
\hline 21 & 30 & 33 & 30 & 27 & 26 & 26 & 25 & 24 & \\
\hline 24 & 55 & 58 & 54 & 53 & 53 & 52 & 51 & 51 & \\
\hline 27 & 45 & 47 & 45 & 44 & 43 & 43 & 42 & 42 & \\
\hline 30 & 27 & 29 & 27 & 23 & 23 & 23 & 23 & 21 & \\
\hline 32 & 87 & 89 & 86 & 87 & 87 & 87 & 87 & 87 & \\
\hline Av. & 35.2 & 34.6 & 34.6 & 34.7 & 35.2 & 35.2 & 34.0 & 34.2 & \\
\hline
\end{tabular}

Table 3: Powers of 5\% tests under symmetric alternatives based on 100000 simulations using empirical critical values with Av. $\geq 34$. 


\begin{tabular}{|c|c|c|c|c|c|c|c|c|c|}
\hline \multicolumn{10}{|c|}{$n=20$} \\
\hline \multicolumn{10}{|c|}{ skew } \\
\hline$k$ & 1 & \multicolumn{5}{|c|}{2} & \multicolumn{3}{|c|}{3} \\
\hline Tests & $\hat{T}_{n}^{(r, k)}$ & $\hat{T}_{n}^{(r, k)}$ & $\hat{T}_{n}^{(r, k)}$ & $\hat{T}_{n}^{(r, k)}$ & $\hat{T}_{n}^{(r, k)}$ & $\hat{T}_{n}^{(r, k)}$ & $\hat{T}_{n}^{(r, k)}$ & $\hat{T}_{n ; c)}^{(r, k)}$ & $\hat{T}_{n}^{(r, k)}$ \\
\hline Alt.M-S/r & 0.5 & 1.0 & 1.1 & 1.3 & 1.5 & 1.7 & 0.1 & 0.3 & 0.3 \\
\hline 36 & 58 & 56 & 54 & 49 & $\overline{43}$ & 37 & 51 & 56 & 45 \\
\hline 38 & 14 & 12 & 12 & 13 & 15 & 16 & 24 & 17 & 33 \\
\hline 40 & 30 & 30 & 32 & 32 & 31 & 29 & 31 & 40 & 29 \\
\hline 42 & 86 & 79 & 83 & 85 & 85 & 83 & 78 & 81 & 77 \\
\hline 44 & 16 & 17 & 18 & 19 & 20 & 19 & 16 & 22 & 16 \\
\hline 50 & 53 & 55 & 56 & 56 & 56 & 54 & 55 & 63 & 54 \\
\hline 52 & 58 & 54 & 56 & 58 & 59 & 60 & 51 & 44 & 52 \\
\hline 54 & 66 & 61 & 64 & 66 & 66 & 66 & 65 & 59 & 65 \\
\hline 56 & 100 & 95 & 97 & 99 & 99 & 99 & 100 & 98 & 100 \\
\hline 58 & 92 & 92 & 92 & 92 & 91 & 90 & 93 & 95 & 93 \\
\hline Av. & 57.2 & 55.0 & 56.6 & 56.9 & 56.5 & 55.2 & 56.3 & 57.4 & 56.3 \\
\hline$k$ & 3 & \multicolumn{4}{|c|}{4} & \multicolumn{4}{|c|}{5} \\
\hline Tests & $\hat{T}_{n ; c_{2}}^{(r, k)}$ & $\hat{T}_{n}^{(r, k)}$ & $\hat{T}_{n ; c_{2}}^{(r, k)}$ & $\hat{T}_{n}^{(r, k)}$ & $\hat{T}_{n ; c_{2}}^{(r, k)}$ & $\hat{T}_{n}^{(r, k)}$ & $\hat{T}_{n}^{(r, k)}$ & $\hat{T}_{n ; c_{2}}^{(r, k)}$ & $\hat{T}_{n ; c_{2}}^{(r, k)}$ \\
\hline Alt.M-S/r & 0.5 & 0.3 & 0.5 & 0.5 & 0.7 & 0.3 & 0.5 & 0.7 & 0.9 \\
\hline 36 & 41 & 57 & 64 & 52 & 51 & 62 & 60 & 68 & 58 \\
\hline 38 & 27 & 28 & 17 & 32 & 24 & 24 & 27 & 16 & 22 \\
\hline 40 & 30 & 31 & 41 & 29 & 32 & 31 & 31 & 40 & 33 \\
\hline 42 & 78 & 78 & 82 & 77 & 81 & 75 & 76 & 80 & 80 \\
\hline 44 & 18 & 15 & 21 & 14 & 17 & 14 & 15 & 19 & 17 \\
\hline 50 & 55 & 55 & 62 & 52 & 56 & 54 & 55 & 61 & 56 \\
\hline 52 & 53 & 47 & 42 & 47 & 48 & 44 & 44 & 42 & 45 \\
\hline 54 & 65 & 64 & 59 & 64 & 63 & 63 & 62 & 59 & 62 \\
\hline 56 & 99 & 100 & 99 & 100 & 100 & 100 & 100 & 99 & 100 \\
\hline 58 & 92 & 94 & 95 & 93 & 94 & 94 & 94 & 96 & 94 \\
\hline Av. & 55.9 & 56.7 & 58.2 & 55.8 & 56.6 & 56.1 & 56.4 & 58.0 & 56.7 \\
\hline
\end{tabular}

Table 4: Powers of 5\% tests under skew alternatives based on 100000 simulations using empirical critical values with Av. $\geq 55$. 


\begin{tabular}{|c|c|c|c|c|c|c|c|c|}
\hline \multicolumn{9}{|c|}{$n=20$} \\
\hline \multicolumn{9}{|c|}{ symmetric } \\
\hline$k$ & \multicolumn{2}{|c|}{1} & \multicolumn{3}{|c|}{2} & \multicolumn{3}{|c|}{3} \\
\hline Tests & $\hat{T}_{n ; c_{4}}^{(r, k)}$ & $\hat{T}_{n ; c_{2}}^{(r, k)}$ & $\hat{T}_{n}^{(r, k)}$ & $\hat{T}_{n ; c_{2}}^{(r, k)}$ & $\hat{T}_{n ; c_{2}}^{(r, k)}$ & $\hat{T}_{n ; c_{4}}^{(r, k)}$ & $\hat{T}_{n}^{(r, k)}$ & $\hat{T}_{n ; c_{4}}^{(r, k)}$ \\
\hline Alt.K-L/r & 0.3 & 1.0 & 0.5 & 0.7 & 0.9 & 0.9 & 0.9 & 1.0 \\
\hline 1 & 43 & 42 & 34 & 41 & 38 & 49 & 46 & 51 \\
\hline 2 & 28 & 28 & 19 & 25 & 23 & 36 & 31 & 37 \\
\hline 5 & 15 & 15 & 9 & 13 & 11 & 23 & 18 & 23 \\
\hline 15 & 13 & 13 & 14 & 14 & 13 & 9 & 10 & 9 \\
\hline 17 & 58 & 56 & 71 & 68 & 68 & 69 & 76 & 71 \\
\hline 20 & 21 & 21 & 21 & 20 & 20 & 15 & 18 & 15 \\
\hline 22 & 71 & 70 & 74 & 71 & 69 & 65 & 71 & 63 \\
\hline 25 & 38 & 38 & 38 & 36 & 36 & 32 & 34 & 31 \\
\hline 27 & 46 & 46 & 47 & 45 & 45 & 41 & 44 & 41 \\
\hline 28 & 45 & 45 & 48 & 47 & 47 & 37 & 43 & 37 \\
\hline Av. & 37.9 & 37.6 & 37.5 & 38.0 & 37.0 & 37.5 & 39.0 & 37.8 \\
\hline$k$ & \multicolumn{6}{|c|}{3} & \multicolumn{2}{|c|}{4} \\
\hline Tests & $\hat{T}_{n}^{(r, k)}$ & $\hat{T}_{n ; c_{4}}^{(r, k)}$ & $\hat{T}_{n}^{(r, k)}$ & $\hat{T}_{n ; c_{2}}^{(r, k)}$ & $\hat{T}_{n}^{(r, k)}$ & $\hat{T}_{n ; c_{2}}^{(r, k)}$ & $\hat{T}_{n}^{(r, k)}$ & $\hat{T}_{n}^{(r, k)}$ \\
\hline Alt.K-L/r & 1.0 & 1.1 & 1.1 & 1.3 & 1.3 & 1.5 & 1.3 & 1.5 \\
\hline 1 & 50 & 51 & 51 & 44 & 49 & 44 & 46 & 51 \\
\hline 2 & 34 & 37 & 34 & 29 & 32 & 29 & 33 & 36 \\
\hline 5 & 19 & 23 & 19 & 17 & 19 & 17 & 21 & 22 \\
\hline 15 & 11 & 9 & 10 & 11 & 10 & 11 & 9 & 9 \\
\hline 17 & 78 & 72 & 78 & 75 & 78 & 77 & 76 & 78 \\
\hline 20 & 17 & 15 & 17 & 16 & 16 & 15 & 15 & 15 \\
\hline 22 & 70 & 62 & 70 & 69 & 69 & 65 & 66 & 64 \\
\hline 25 & 34 & 30 & 33 & 33 & 33 & 31 & 31 & 30 \\
\hline 27 & 44 & 40 & 43 & 42 & 42 & 40 & 42 & 41 \\
\hline 28 & 43 & 36 & 42 & 42 & 41 & 41 & 37 & 37 \\
\hline Av. & 40,1 & 37,4 & 39,8 & 37,9 & 38,9 & 37,1 & 37,5 & 38,3 \\
\hline
\end{tabular}

Table 5: Powers of 5\% tests under symmetric alternatives based on 100000 simulations using empirical critical values with Av. $\geq 37$. 


\begin{tabular}{|c|c|c|c|c|c|c|c|c|c|c|}
\hline \multicolumn{11}{|c|}{$n=20$} \\
\hline \multicolumn{11}{|c|}{ skew } \\
\hline$k$ & \multicolumn{4}{|c|}{1} & \multicolumn{6}{|c|}{2} \\
\hline Tests & $\hat{T}_{n}^{(r, k)}$ & $\hat{T}_{n}^{(r, k)}$ & $\hat{T}_{n}^{(r, k)}$ & $\hat{T}_{n}^{(r, k)}$ & $\hat{T}_{n ; c_{3}}^{(r, k)}$ & $\hat{T}_{n}^{(r, k)}$ & $\hat{T}_{n ; c_{3}}^{(r, k)}$ & $\hat{T}_{n ; c_{1}}^{(r, k)}$ & $\hat{T}_{n}^{(r, k)}$ & $\hat{T}_{n ; c_{1}}^{(r, k)}$ \\
\hline Alt.K-L/r & 0.5 & 1.5 & 1.7 & 2.0 & 0.1 & 0.1 & 0.3 & 1.0 & 1.0 & 1.1 \\
\hline 36 & 59 & 38 & 35 & 30 & 34 & 32 & 26 & 38 & 56 & 33 \\
\hline 40 & 30 & 25 & 25 & 26 & 36 & 29 & 31 & 37 & 31 & 35 \\
\hline 41 & 27 & 25 & 26 & 28 & 33 & 29 & 31 & 33 & 27 & 32 \\
\hline 44 & 16 & 16 & 16 & 17 & 23 & 19 & 22 & 23 & 17 & 23 \\
\hline 45 & 27 & 29 & 30 & 32 & 33 & 32 & 35 & 31 & 28 & 33 \\
\hline 46 & 26 & 26 & 27 & 29 & 35 & 31 & 34 & 34 & 28 & 34 \\
\hline 47 & 21 & 22 & 23 & 24 & 24 & 24 & 26 & 21 & 22 & 23 \\
\hline 48 & 78 & 79 & 79 & 80 & 72 & 78 & 74 & 67 & 71 & 71 \\
\hline 49 & 23 & 24 & 25 & 26 & 27 & 27 & 28 & 25 & 25 & 27 \\
\hline 50 & 53 & 51 & 52 & 53 & 61 & 56 & 58 & 62 & 55 & 61 \\
\hline 52 & 57 & 58 & 59 & 59 & 51 & 58 & 56 & 46 & 54 & 51 \\
\hline 54 & 66 & 66 & 66 & 66 & 60 & 65 & 64 & 51 & 61 & 60 \\
\hline 57 & 71 & 72 & 72 & 70 & 63 & 66 & 56 & 65 & 69 & 62 \\
\hline 58 & 92 & 90 & 90 & 90 & 90 & 92 & 90 & 85 & 91 & 89 \\
\hline Av. & 46.2 & 44.2 & 44.7 & 45.0 & 45.8 & 45.6 & 45.1 & 44.2 & 45.3 & 45.3 \\
\hline$k$ & \multicolumn{6}{|c|}{2} & \multicolumn{2}{|c|}{3} & 4 & 5 \\
\hline Tests & $\hat{T}_{n}^{(r, k)}$ & $\hat{T}_{n ; c_{1}}^{(r, k)}$ & $\hat{T}_{n}^{(r, k)}$ & $\hat{T}_{n}^{(r, k)}$ & $\hat{T}_{n}^{(r, k)}$ & $\hat{T}_{n}^{(r, k)}$ & $\hat{T}_{n}^{(r, k)}$ & $\begin{array}{l}\hat{T}_{n ; c_{2}}^{(r, k)} \\
\end{array}$ & $\hat{T}_{n ; c_{2}}^{(r, k)}$ & $\hat{T}_{n ; c_{2}}^{(r, k)}$ \\
\hline Alt.K-L/r & 1.1 & 1.3 & 1.3 & 1.5 & 1.7 & 2.0 & 0.1 & 0.3 & 0.5 & 0.7 \\
\hline 36 & 54 & 26 & 49 & 43 & 38 & 29 & 51 & 57 & 63 & 68 \\
\hline 40 & 32 & 31 & 31 & 31 & 29 & 25 & 31 & 41 & 40 & 40 \\
\hline 41 & 29 & 31 & 31 & 32 & 33 & 31 & 26 & 34 & 31 & 29 \\
\hline 44 & 18 & 22 & 19 & 20 & 19 & 19 & 16 & 22 & 20 & 20 \\
\hline 45 & 30 & 35 & 32 & 34 & 35 & 36 & 25 & 28 & 24 & 23 \\
\hline 46 & 29 & 34 & 30 & 30 & 31 & 30 & 26 & 32 & 29 & 29 \\
\hline 47 & 23 & 25 & 23 & 25 & 26 & 27 & 17 & 18 & 16 & 16 \\
\hline 48 & 75 & 74 & 78 & 81 & 82 & 81 & 73 & 68 & 65 & 64 \\
\hline 49 & 25 & 28 & 26 & 27 & 28 & 28 & 21 & 23 & 20 & 20 \\
\hline 50 & 56 & 57 & 56 & 56 & 54 & 51 & 56 & 63 & 61 & 62 \\
\hline 52 & 56 & 57 & 58 & 59 & 60 & 61 & 51 & 44 & 42 & 42 \\
\hline 54 & 64 & 64 & 66 & 66 & 66 & 66 & 65 & 58 & 59 & 59 \\
\hline 57 & 69 & 56 & 69 & 67 & 64 & 54 & 74 & 64 & 58 & 54 \\
\hline 58 & 92 & 90 & 92 & 91 & 90 & 88 & 94 & 95 & 95 & 96 \\
\hline Av. & 46.5 & 45.0 & 47.1 & 47.3 & 46.7 & 44.7 & 44.7 & 46.3 & 44.5 & 44.4 \\
\hline
\end{tabular}

Table 6: Powers of 5\% tests under skew alternatives based on 100000 simulations using empirical critical values with Av. $\geq 44$. 


\begin{tabular}{|c|c|c|c|c|c|c|c|c|}
\hline \multicolumn{9}{|c|}{$n=50$} \\
\hline \multicolumn{9}{|c|}{ symmetric } \\
\hline$k$ & \multicolumn{5}{|c|}{1} & \multicolumn{3}{|c|}{2} \\
\hline Tests & $\hat{T}_{n ; c_{2}}^{(r, k)}$ & $\hat{T}_{n ; c_{4}}^{(r, k)}$ & $\hat{T}_{n ; c_{2}}^{(r, k)}$ & $\hat{T}_{n ; c_{2}}^{(r, k)}$ & $\hat{T}_{n ; c_{2}}^{(r, k)}$ & $\hat{T}_{n ; c_{4}}^{(r, k)}$ & $\hat{T}_{n ; c_{4}}^{(r, k)}$ & $\hat{T}_{n}^{(r, k)}$ \\
\hline Alt.M-S/r & 0.3 & 0.3 & 0.9 & 1.0 & 1.1 & 0.3 & 0.5 & 0.5 \\
\hline 1 & 88 & 95 & 92 & 93 & 85 & 93 & 94 & 95 \\
\hline 4 & 59 & 67 & 60 & 62 & 56 & 77 & 72 & 59 \\
\hline 7 & 21 & 23 & 19 & 20 & 19 & 38 & 31 & 16 \\
\hline 15 & 24 & 25 & 25 & 25 & 24 & 18 & 19 & 24 \\
\hline 18 & 57 & 59 & 57 & 58 & 57 & 53 & 54 & 61 \\
\hline 21 & 61 & 62 & 61 & 62 & 62 & 54 & 52 & 59 \\
\hline 24 & 89 & 89 & 89 & 89 & 89 & 86 & 85 & 88 \\
\hline 27 & 78 & 78 & 78 & 78 & 78 & 76 & 75 & 77 \\
\hline 30 & 53 & 55 & 53 & 54 & 53 & 47 & 46 & 53 \\
\hline 32 & 100 & 100 & 100 & 100 & 100 & 100 & 100 & 100 \\
\hline Av. & 62.9 & 65.5 & 63.3 & 64.0 & 62.3 & 64.0 & 62.8 & 63.2 \\
\hline$k$ & \multicolumn{4}{|c|}{2} & \multicolumn{4}{|c|}{3} \\
\hline Tests & $\hat{T}_{n ; c_{2}}^{(r, k)}$ & $\hat{T}_{n}^{(r, k)}$ & $\hat{T}_{n ; c_{2}}^{(r, k)}$ & $\hat{T}_{n ; c_{2}}^{(r, k)}$ & $\hat{T}_{n ; c_{4}}^{(r, k)}$ & $\hat{T}_{n ; c_{4}}^{(r, k)}$ & $\hat{T}_{n ; c_{2}}^{(r, k)}$ & $\hat{T}_{n ; c_{2}}^{(r, k)}$ \\
\hline Alt.M-S/r & 0.7 & 0.7 & 0.9 & 1.0 & 0.9 & 1.0 & 1.3 & 1.5 \\
\hline 1 & 94 & 93 & 95 & 94 & 95 & 95 & 93 & 94 \\
\hline 4 & 63 & 53 & 62 & 59 & 72 & 71 & 61 & 62 \\
\hline 7 & 21 & 14 & 20 & 19 & 32 & 31 & 23 & 23 \\
\hline 15 & 25 & 24 & 25 & 25 & 18 & 18 & 21 & 21 \\
\hline 18 & 63 & 61 & 64 & 63 & 56 & 56 & 64 & 65 \\
\hline 21 & 58 & 59 & 58 & 58 & 49 & 48 & 52 & 51 \\
\hline 24 & 87 & 88 & 87 & 87 & 84 & 83 & 85 & 84 \\
\hline 27 & 76 & 77 & 76 & 76 & 73 & 72 & 74 & 73 \\
\hline 30 & 54 & 53 & 54 & 54 & 45 & 45 & 50 & 50 \\
\hline 32 & 100 & 100 & 100 & 99 & 100 & 100 & 100 & 100 \\
\hline Av. & 64.2 & 62.2 & 64.3 & 63.4 & 62.4 & 62.0 & 62.2 & 62.2 \\
\hline
\end{tabular}

Table 7: Powers of 5\% tests under symmetric alternatives based on 100000 simulations using empirical critical values with Av. $\geq 62$. 


\begin{tabular}{|c|c|c|c|c|c|c|c|c|c|}
\hline \multicolumn{10}{|c|}{$n=50$} \\
\hline \multicolumn{10}{|c|}{ skew } \\
\hline$k$ & \multicolumn{6}{|c|}{2} & \multicolumn{3}{|c|}{3} \\
\hline Tests & $\hat{T}_{n}^{(r, k)}$ & $\hat{T}_{n}^{(r, k)}$ & $\hat{T}_{n}^{(r, k)}$ & $\hat{T}_{n}^{(r, k)}$ & $\hat{T}_{n}^{(r, k)}$ & $\hat{T}_{n}^{(r, k)}$ & $\hat{T}_{n}^{(r, k)}$ & $\hat{T}_{n}^{(r, k)}$ & $\hat{T}_{n}^{(r, k)}$ \\
\hline Alt.M-S/r & 0.7 & 0.9 & 1.0 & 1.1 & 1.3 & 1.5 & 0.1 & 0.3 & 1.3 \\
\hline 36 & 98 & 98 & 98 & 97 & 95 & 92 & 94 & 84 & 99 \\
\hline 38 & 68 & 65 & 64 & 63 & 64 & 66 & 70 & 78 & 63 \\
\hline 40 & 71 & 74 & 73 & 73 & 69 & 65 & 80 & 71 & 77 \\
\hline 42 & 93 & 100 & 100 & 100 & 100 & 100 & 100 & 99 & 98 \\
\hline 44 & 36 & 40 & 40 & 40 & 39 & 38 & 45 & 38 & 37 \\
\hline 50 & 92 & 93 & 93 & 93 & 92 & 90 & 95 & 93 & 91 \\
\hline 52 & 87 & 88 & 88 & 88 & 89 & 89 & 85 & 84 & 79 \\
\hline 54 & 92 & 92 & 92 & 93 & 93 & 93 & 92 & 92 & 91 \\
\hline 56 & 100 & 100 & 100 & 100 & 100 & 100 & 100 & 100 & 100 \\
\hline 58 & 100 & 100 & 100 & 100 & 100 & 100 & 100 & 100 & 100 \\
\hline Av. & 83.8 & 84.9 & 84.8 & 84.7 & 84.0 & 83.2 & 86.2 & 84.0 & 83.6 \\
\hline$k$ & \multicolumn{3}{|c|}{3} & \multicolumn{3}{|c|}{4} & \multicolumn{3}{|c|}{5} \\
\hline Tests & $\hat{T}_{n}^{(r, k)}$ & $\hat{T}_{n}^{(r, k)}$ & $\hat{T}_{n}^{(r, k)}$ & $\hat{T}_{n}^{(r, k)}$ & $\hat{T}_{n}^{(r, k)}$ & $\hat{T}_{n}^{(r, k)}$ & $\hat{T}_{n}^{(r, k)}$ & $\hat{T}_{n}^{(r, k)}$ & $\hat{T}_{n}^{(r, k)}$ \\
\hline Alt.M-S/r & 1.5 & 1.7 & 2.0 & 0.1 & 0.3 & 0.5 & 0.1 & 0.3 & 0.5 \\
\hline 36 & 99 & 98 & 97 & 99 & 97 & 93 & 99 & 99 & 98 \\
\hline 38 & 61 & 60 & 60 & 71 & 74 & 73 & 71 & 71 & 70 \\
\hline 40 & 78 & 77 & 74 & 82 & 79 & 73 & 82 & 82 & 78 \\
\hline 42 & 100 & 100 & 100 & 100 & 100 & 100 & 100 & 100 & 100 \\
\hline 44 & 40 & 41 & 40 & 44 & 41 & 36 & 41 & 43 & 39 \\
\hline 50 & 92 & 92 & 92 & 96 & 95 & 93 & 95 & 96 & 94 \\
\hline 52 & 79 & 80 & 80 & 83 & 81 & 80 & 80 & 80 & 78 \\
\hline 54 & 91 & 91 & 91 & 92 & 92 & 92 & 92 & 92 & 91 \\
\hline 56 & 100 & 100 & 100 & 100 & 100 & 100 & 100 & 100 & 100 \\
\hline 58 & 100 & 100 & 100 & 100 & 100 & 100 & 100 & 100 & 100 \\
\hline Av. & 84.0 & 83.9 & 83.3 & 86.6 & 85.9 & 83.9 & 86.0 & 86.2 & 84.7 \\
\hline
\end{tabular}

Table 8: Powers of 5\% tests under skew alternatives based on 100000 simulations using empirical critical values with Av. $\geq 83$. 


\begin{tabular}{|c|c|c|c|c|c|c|c|c|c|c|}
\hline \multicolumn{11}{|c|}{$n=50$} \\
\hline \multicolumn{11}{|c|}{ symmetric } \\
\hline$k$ & & 1 & & & & & 2 & & & \\
\hline Tests & $\hat{T}_{n ; c_{4}}^{(r, k)}$ & $\hat{T}_{n ; c 2}^{(r, k)}$ & $\hat{T}_{n ; c_{2}}^{(r, k)}$ & $\hat{T}_{n ; c_{4}}^{(r, k)}$ & $\hat{T}_{n ; c_{4}}^{(r, k)}$ & $\hat{T}_{n}^{(r, k)}$ & $\hat{T}_{n ; c_{2}}^{(r, k)}$ & $\hat{T}_{n}^{(r, k)}$ & $\hat{T}_{n ; c_{2}}^{(r, k)}$ & $\hat{T}_{n ; c_{2}}^{(r, k)}$ \\
\hline Alt.K-L/r & 0.3 & 0.9 & 1.0 & 0.3 & 0.5 & 0.5 & 0.7 & 0.7 & 0.9 & 1.0 \\
\hline 1 & 95 & 92 & 93 & 93 & 94 & 95 & 94 & 93 & 95 & 94 \\
\hline 2 & 83 & 79 & 80 & 87 & 86 & 80 & 81 & 76 & 82 & 79 \\
\hline 5 & 57 & 51 & 53 & 70 & 64 & 48 & 52 & 43 & 52 & 50 \\
\hline 15 & 25 & 24 & 25 & 18 & 19 & 24 & 24 & 24 & 24 & 25 \\
\hline 17 & 91 & 87 & 88 & 98 & 97 & 97 & 95 & 96 & 94 & 92 \\
\hline 20 & 42 & 42 & 42 & 36 & 34 & 39 & 38 & 39 & 38 & 38 \\
\hline 22 & 98 & 98 & 98 & 97 & 97 & 98 & 98 & 98 & 97 & 96 \\
\hline 25 & 69 & 68 & 68 & 64 & 63 & 67 & 65 & 66 & 66 & 65 \\
\hline 27 & 78 & 78 & 78 & 75 & 75 & 77 & 76 & 77 & 76 & 76 \\
\hline 28 & 82 & 80 & 81 & 78 & 77 & 82 & 83 & 82 & 83 & 82 \\
\hline Av. & 72.0 & 69.8 & 70.6 & 71.7 & 70.5 & 70.8 & 70.7 & 69.4 & 70.7 & 69.8 \\
\hline$k$ & & & & & 3 & & & & & \\
\hline Tests & $\hat{T}_{n ; c_{3}}^{(r, k)}$ & $\hat{T}_{n}^{(r, k)}$ & $\hat{T}_{n ; c_{1}}^{(r, k)}$ & $\hat{T}_{n ; c_{4}}^{(r, k)}$ & $\hat{T}_{n}^{(r, k)}$ & $\hat{T}_{n ; c_{4}}^{(r, k)}$ & $\hat{T}_{n}^{(r, k)}$ & $\hat{T}_{n ; c_{4}}^{(r, k)}$ & $\hat{T}_{n}^{(r, k)}$ & \\
\hline Alt.K-L/r & -0.499 & -0.499 & 0.5 & 0.9 & 0.9 & 1.0 & 1.0 & 1.1 & 1.1 & \\
\hline 1 & 90 & 90 & 90 & 95 & 95 & 96 & 95 & 95 & 95 & \\
\hline 2 & 86 & 86 & 86 & 85 & 82 & 85 & 81 & 85 & 80 & \\
\hline 5 & 70 & 70 & 70 & 63 & 54 & 63 & 53 & 62 & 52 & \\
\hline 15 & 15 & 15 & 15 & 18 & 20 & 18 & 20 & 18 & 20 & \\
\hline 17 & 98 & 98 & 98 & 99 & 99 & 99 & 99 & 99 & 99 & \\
\hline 20 & 30 & 30 & 30 & 31 & 33 & 30 & 33 & 29 & 32 & \\
\hline 22 & 96 & 96 & 96 & 97 & 97 & 97 & 97 & 96 & 97 & \\
\hline 25 & 60 & 60 & 60 & 61 & 62 & 60 & 62 & 59 & 62 & \\
\hline 27 & 73 & 73 & 73 & 73 & 74 & 72 & 74 & 72 & 74 & \\
\hline 28 & 73 & 73 & 73 & 78 & 79 & 77 & 79 & 77 & 80 & \\
\hline Av. & 69.1 & 69.1 & 69.0 & 69.9 & 69.6 & 69.7 & 69.4 & 69.1 & 69.1 & \\
\hline
\end{tabular}

Table 9: Powers of 5\% tests under symmetric alternatives based on 100000 simulations using empirical critical values with Av. $\geq 69$. 


\begin{tabular}{|c|c|c|c|c|c|c|c|c|}
\hline \multicolumn{9}{|c|}{$n=50$} \\
\hline \multicolumn{9}{|c|}{ skew } \\
\hline$k$ & \multicolumn{4}{|c|}{1} & \multicolumn{4}{|c|}{2} \\
\hline Tests & $\hat{T}_{n}^{(r, k)}$ & $\hat{T}_{n}^{(r, k)}$ & $\hat{T}_{n}^{(r, k)}$ & $\hat{T}_{n}^{(r, k)}$ & $\hat{T}_{n ; c 3}^{(r, k)}$ & $\hat{T}_{n}^{(r, k)}$ & $\hat{T}_{n}^{(r, k)}$ & $\hat{T}_{n}^{(r, k)}$ \\
\hline Alt.K-L/r & 0.5 & 1.3 & 1.5 & 1.7 & 0.1 & 0.7 & 0.9 & 1.0 \\
\hline 36 & 97 & 92 & 87 & 80 & 62 & 99 & 98 & 98 \\
\hline 40 & 75 & 65 & 66 & 65 & 72 & 72 & 74 & 74 \\
\hline 41 & 62 & 56 & 60 & 62 & 69 & 52 & 60 & 63 \\
\hline 44 & 41 & 36 & 38 & 39 & 48 & 37 & 40 & 41 \\
\hline 45 & 58 & 58 & 60 & 63 & 64 & 55 & 57 & 58 \\
\hline 46 & 60 & 57 & 59 & 60 & 68 & 56 & 60 & 60 \\
\hline 47 & 45 & 46 & 46 & 48 & 41 & 42 & 42 & 43 \\
\hline 48 & 99 & 99 & 99 & 99 & 97 & 97 & 98 & 98 \\
\hline 49 & 47 & 47 & 48 & 50 & 49 & 46 & 47 & 48 \\
\hline 50 & 93 & 91 & 91 & 92 & 94 & 92 & 93 & 93 \\
\hline 52 & 90 & 90 & 90 & 90 & 79 & 87 & 88 & 88 \\
\hline 54 & 93 & 93 & 93 & 93 & 88 & 92 & 92 & 93 \\
\hline 57 & 97 & 97 & 97 & 97 & 96 & 97 & 97 & 97 \\
\hline 58 & 100 & 100 & 100 & 100 & 97 & 100 & 100 & 100 \\
\hline Av. & 75.5 & 73.5 & 74.0 & 74.1 & 73.1 & 73.2 & 74.7 & 75.2 \\
\hline$k$ & \multicolumn{4}{|c|}{2} & \multicolumn{2}{|c|}{3} & \multicolumn{2}{|c|}{4} \\
\hline Tests & $\hat{T}_{n}^{(r, k)}$ & $\hat{T}_{n}^{(r, k)}$ & $\hat{T}_{n}^{(r, k)}$ & $\hat{T}_{n}^{(r, k)}$ & $\hat{T}_{n}^{(r, k)}$ & $\hat{T}_{n}^{(r, k)}$ & $\hat{T}_{n}^{(r, k)}$ & $\hat{T}_{n}^{(r, k)}$ \\
\hline Alt.K-L/r & 1.1 & 1.3 & 1.5 & 1.7 & 0.1 & 2.0 & 0.1 & 0.3 \\
\hline 36 & 97 & 95 & 92 & 88 & 93 & 97 & 99 & 97 \\
\hline 40 & 73 & 70 & 65 & 60 & 80 & 74 & 82 & 79 \\
\hline 41 & 65 & 68 & 69 & 68 & 66 & 70 & 61 & 62 \\
\hline 44 & 41 & 40 & 38 & 36 & 45 & 40 & 44 & 41 \\
\hline 45 & 59 & 62 & 64 & 66 & 57 & 55 & 51 & 51 \\
\hline 46 & 61 & 61 & 59 & 58 & 63 & 58 & 61 & 60 \\
\hline 47 & 43 & 45 & 46 & 47 & 36 & 34 & 32 & 31 \\
\hline 48 & 98 & 99 & 99 & 99 & 98 & 97 & 97 & 97 \\
\hline 49 & 48 & 50 & 50 & 51 & 45 & 42 & 41 & 41 \\
\hline 50 & 93 & 92 & 90 & 89 & 95 & 92 & 96 & 95 \\
\hline 52 & 88 & 89 & 89 & 89 & 85 & 81 & 83 & 81 \\
\hline 54 & 93 & 93 & 93 & 93 & 92 & 91 & 92 & 92 \\
\hline 57 & 98 & 98 & 97 & 97 & 98 & 97 & 98 & 97 \\
\hline 58 & 100 & 100 & 100 & 100 & 100 & 100 & 100 & 100 \\
\hline Av. & 75.4 & 75.6 & 75.0 & 74.4 & 75.3 & 73.4 & 74.1 & 73.2 \\
\hline
\end{tabular}

Table 10: Powers of 5\% tests under skew alternatives based on 100000 simulations using empirical critical values with Av. $\geq 73$.

Heaving a look at the above simulations we see that our most powerful tests for $n=20$ are $\hat{T}_{n ; c_{4}}^{(0.3,1)}$ with Av.=36.0 under symmetric Alt.M-S while the standard test $K^{2}$ has Av. $=36.3$ (Table 1 ); $\hat{T}_{n ; c 2}^{(0.5,4)}$ with Av.=58.2 under skew Alt.M-S while the standard test $S$ - $W$ has Av. $=59.6$ (Table 1 ); $\hat{T}_{n}^{(1.0,3)}$ with Av. $=40.1$ under symmetric Alt.K-L while the standard test $W$ has Av. $=39.0$ (Table 2); $\hat{T}_{n}^{(1.5,2)}$ with Av.=47.3 under skew Alt.K-L while the standard test $W$ has Av.=46.9 (Table 2).

The most powerful tests for $n=50$ are: $\hat{T}_{n ; c_{4}}^{(0.3,1)}$ with Av. $=65.5$ under 
symmetric Alt.M-S while the standard test $K^{2}$ has Av. $=61.6$ (Table 1$) ; \hat{T}_{n}^{(0.1,4)}$ with Av. $=86.6$ under skew Alt.M-S while the standard test $S$ - $W$ has Av. $=88.3$ (Table 1$) ; \hat{T}_{n ; c}^{(0.3,1)}$ with Av.=72.0 under symmetric Alt.K-L while the standard test $W$ has Av.=69.5 (Table 2); $\hat{T}_{n}^{(1.3,2)}$ with Av.=75.6 under skew Alt.K-L while the standard test $W$ has Av.=73.1 (Table 2).

Hence we conclude that our tests for normality perform very well and they can be recommended to use them in the statistical inference. Moreover, our simulations show that tests $K^{2}, R$ and $Y$ are poor when alternatives are skew. For skew alternatives the test $S$ - $W$ and ours are best.

\begin{tabular}{|c|c|c|c|c|}
\hline$n \backslash$ Alt. & M-S symmetric & M-S skew & K-L symmetric & K-L skew \\
\hline 20 & $\hat{T}_{20 ; c_{4}}^{(0.3,1)}$ & $\hat{T}_{20 ; c_{2}}^{(0.5,4)}$ & $\hat{T}_{20}^{(1.0,3)}$ & $\hat{T}_{20}^{(1.5,2)}$ \\
& Av. $=36.0$ & Av. $=58.2$ & Av. $=40.1$ & Av. $=47.3$ \\
\hline 50 & $\hat{T}_{50 ; c_{4}}^{(0.3,1)}$ & $\hat{T}_{50}^{(0.1,4)}$ & $\hat{T}_{50 ; c_{4}}^{(0.3,1)}$ & $\hat{T}_{50}^{(1.5,2)}$ \\
& Av. $=65.5$ & Av. $=86.6$ & Av. $=72.0$ & Av. $=75.0$ \\
\hline
\end{tabular}

Table 11: Our simple recommended tests.

It is almost sure that one can find in our family tests better than those presented here choosing different values of $r$ and $k$.

\section{References}

[1] W.C.M. Kallenberg, T. Ledwina, Data driven smooth tests for composite hypotheses: comparison of powers, J. Statist. Comput. Simul., 59 (1997), 101-121.

[2] M. Morris, D. Szynal, Goodness-of-fit tests based on characterizations involving moments of order statistics, J. of Pure and Appl. Math., 38 (2007), 83-121.

[3] M. Morris, D. Szynal, Some $U$-statistics in goodness-of-fit tests derived from characterizations via record values, J. of Pure and Appl. Math., 46 (2008), 507-582.

[4] E.S. Pearson, R.B. D'Agostino, K.O. Bowman, Tests for departure from normality: Comparison of powers, Biometrika, 64, No. 2 (1977), 231-246. 